\title{
SIMULTANEOUS CHEBYSHEV APPROXIMATION OF FUNCTIONS ON AN INTERVAL
}

\author{
CHARLES B. DUNHAM
}

1. Introduction. The following approximation problem is a generalization of the classical problem of Chebyshev approximation of a continuous function on an interval. Let $f^{+}$and $f^{-}$be two continuous functions on a finite interval $[a, b]$ with $f^{+}(x) \leqq f^{-}(x)$. Let $F(A, x)$ be a continuous approximating function with parameter $A$ which is unisolvent of degree $n$ on $[a, b]$. This means for distinct points $x_{1}, \cdots, x_{n}$ of $[a, b]$ and any real numbers $w_{1}, \cdots, w_{n}$, the system of equations

$$
F\left(A, x_{k}\right)=w_{k}, \quad k=1, \cdots, n,
$$

is satisfied by one and only one approximant $F\left(A_{0}, x\right)$. This implies that the difference of two distinct approximants can have at most $n-1$ zeros. For $s$ any superscript define

$$
E^{s}(A, x)=f^{s}(x)-F(A, x)
$$

and for $g$ any function define

$$
\|g\|=\sup \{|g(x)|: a \leqq x \leqq b\} .
$$

The Chebyshev problem of simultaneous approximation of $f^{-}$and $f^{+}$is to choose a parameter $A^{*}$ to minimize

$$
e(A)=\max \left\{\left\|E^{+}(A, x)\right\|,\left\|E^{-}(A, x)\right\|\right\} .
$$

Such a parameter $A^{*}$ and the corresponding approximant $F\left(A^{*}, x\right)$ are called best (to $f^{-}$and $f^{+}$on $[a, b]$ ). In this note we will develop a theory for this problem which is quite close to the classical theory of Chebyshev approximation of one continuous function on an interval, where $f^{-}=f^{+}$. It is sometimes a straightforward matter to extend proofs of standard results in the classical case to proofs of results in the more general case of this note. A good source of standard results is the paper of Tornheim [3], in which unisolvent functions are called $n$-parameter families of functions, or the book of Rice [2].

For completeness we will in fact consider the more general case where $f^{+}$is upper semicontinuous [4], that is $\left\{x: f^{+}(x) \geqq r\right\}$ is closed for all real $r$ and $f^{-}$is lower semicontinuous, that is, $\left\{x: f^{-}(x) \leqq r\right\}$ is closed for all real $r$. This ensures that $f^{+}(x)-F(A, x)$ attains its supremum on compact sets and $f^{-}(x)-F(A, x)$ attains its infimum on

Received by the editors June 30, 1966. 
compact sets. Hence for any parameter $A$ there is at least one point of $\lfloor a, b]$ at which $f^{+}(x)-F(A, x)$ attains $e(A)$ or $f^{-}(x)-F(A, x)$ attains $-e(A)$. The proofs of this note require only slight modifications to make them applicable to the more general approximating functions unisolvent of variable degree [1].

The author wishes to thank Professor E. Barbeau for his careful criticism of this note.

2. Characterization of best approximations. Examination of the existence proof for the classical case [3, p. 464] shows that it holds for the case of approximation of bounded $f^{-}$and $f^{+}$also. We now study the problem of characterizing best approximations. Let us suppose that for some point $x_{0}$ of $[a, b]$ and some parameter $A$,

$$
f^{+}\left(x_{0}\right)-F\left(A, x_{0}\right)=F\left(A, x_{0}\right)-f^{-}\left(x_{0}\right)=e(A) .
$$

Clearly, $F(A, x)$ is a best approximation to $f^{-}$and $f^{+}$, as no approximant can make both errors smaller at the point $x_{0}$. Furthermore, any best parameter must satisfy (3). We will therefore define a point $x_{0}$ to be a straddle point in the case of approximation of $f^{-}$and $f^{+}$if there exists a parameter $A$ for which (3) is satisfied. In the classical case of approximation of a single continuous function, a straddle point occurs if and only if the function being approximated is an approximant, and in this case all points are straddle points.

We will require symbols to denote the signs + and - . The symbol $\rho(i)$ will stand for + if $i$ is even and - if $i$ is odd. The symbol $\sigma$ will denote a sign which may be + or - .

An approximation $F\left(A_{0}, x\right)$ will be said to have $n$ alternations on an interval $[a, b]$ if there exists an ordered $n+1$ point set $\left\{x_{0}, \cdots, x_{n}\right\}$, $a \leqq x_{0}<\cdots<x_{n} \leqq b$, and an integer $i=0$ or 1 such that

$$
E^{\rho(i+k)}\left(A_{0}, x_{k}\right)=(-1)^{i+k} e\left(A_{0}\right), \quad k=0, \cdots, n .
$$

We now give a generalization of the de la Vallée Poussin lemma.

Lemma 1. Suppose for $i=0$ or $1, E^{\rho(i+k)}\left(A_{0}, x_{k}\right)$ alternates in sign on an ordered $n+1$ point set $\left\{x_{0}, \cdots, x_{n}\right\}$, then if $A \neq A_{0}$,

$$
\begin{aligned}
\max \left\{\mid E^{\rho(i+k)}\right. & \left.\left(A, x_{k}\right) \mid: k=0, \cdots, n\right\} \\
& \geqq \min \left\{\left|E^{\rho(i+k)}\left(A_{0}, x_{k}\right)\right|: k=0, \cdots, n\right\}
\end{aligned}
$$

Proof. If the inequality did not hold then,

$$
\left|f^{\rho(i+k)}-F\left(A, x_{k}\right)\right|<\left|f^{\rho(i+k)}-F\left(A_{0}, x_{k}\right)\right|, \quad k=0, \cdots, n .
$$

Hence $F\left(A_{0}, x_{k}\right)-F\left(A, x_{k}\right)$ alternates in sign on the ordered point set $\left\{x_{0}, \cdots, x_{n}\right\}$ and the continuous function $F\left(A_{0}, x\right)-F(A, x)$ must 
have $n$ zeros on the interval $[a, b]$. This contradicts the unisolvence condition, proving the lemma.

Theorem 1. $F\left(A^{*}, x\right)$ is a best approximation to $f^{-}$and $f^{+}$on $[a, b]$ if and only if $F\left(A^{*}, x\right)$ has a straddle point or $n$ alternations on $[a, b]$.

Proof. Sufficiency follows from the above lemma and the remark on straddle points. We now examine necessity. Let $F\left(A^{*}, x\right)$ be an approximation with no straddle points. With $\sigma$ standing for the signs + or - , we define a point $x$ of $[a, b]$ to be a $\sigma$ point if $E^{\sigma}\left(A^{*}, x\right)$ $=\sigma e\left(A^{*}\right)$, thus defining + points and - points. Define $M^{\sigma}$ to be the set of $\sigma$ points, then because $\sigma E^{\sigma}\left(A^{*}, x\right)=\sigma\left(f^{\sigma}(x)-F\left(A^{*}, x\right)\right)$ is upper semicontinuous, the set $M^{\sigma}$ is closed. Let us define

$$
u=\inf \left\{\left|x_{1}-x_{2}\right|: x_{1} \in M^{-}, x_{2} \in M^{+}\right\} .
$$

Now $\left|x_{1}-x_{2}\right|$ is a continuous function on the compact space $M^{-} \times M^{+}$ and so attains its infimum for $x_{1} \in M^{-}, x_{2} \in M^{+}$. If that infimum is zero, then there is a point $x$ which is both a + point and a - point. Such a point is a straddle point and by hypothesis $F\left(A^{*}, x\right)$ has no straddle points. Hence $\mu$ must be positive. Define

$$
V_{\eta}^{\sigma}=\left\{x:\left|x-x_{1}\right|<\eta, a \leqq x \leqq b, x_{1} \in M^{\sigma}\right\} .
$$

Let $C(T)$ denote the closure of a set $T$ and define $H^{+}=C\left(V_{\mu / 3}^{+}\right) \sim V_{\mu / 6}^{+}$. Since $f^{+}$is the infimum of a nonvoid family of continuous functions on $[a, b]$ and $f^{+}(x)<F\left(A^{*}, x\right)+e\left(A^{*}\right)$, for any $y \in H^{+}$there exists a continuous function $h_{y}$ such that $h_{y}(y)<F\left(A^{*}, y\right)+e\left(A^{*}\right), h_{y} \geqq f^{+}$. Since $h_{y}$ and $F\left(A^{*}, x\right)$ are continuous, $h_{y}(x)<F\left(A^{*}, x\right)+e\left(A^{*}\right)$ on a neighbourhood $U_{y}$ of $y$. Cover $H^{+}$by a finite set $S$ of such neighbourhoods $U_{\nu}$ and let $h_{\nu}$ be the corresponding functions. On $C\left(V_{\mu / 3}^{+}\right)$define

$$
f(x)=\inf \left[\left\{h_{\nu}(x): U_{\nu} \in S\right\} \cup\left\{F\left(A^{*}, x\right)+e\left(A^{*}\right)\right\}\right],
$$

then $f$ is continuous on $C\left(V_{\mu / 3}^{+}\right)$, being the infimum of a finite number of continuous functions, and

$$
\begin{array}{rlrl}
f(x) \geqq f^{+}(x), & & x \in C\left(V_{\mu / 3}^{+}\right), \\
f(x) & =f^{+}(x), & & x \in M^{+}, \\
E\left(A^{*}, x\right) & <e\left(A^{*}\right), & & x \in V_{\mu / 6}^{+} .
\end{array}
$$

Similarly on $C\left(V_{\mu / 3}^{-}\right)$it is possible to define the function $f$ so that

$$
\begin{array}{rlrl}
f(x) \leqq f^{-}(x), & & x \in C\left(V_{\mu / 3}^{-}\right), \\
f(x)=f^{-}(x), & & x \in M^{-}, \\
E\left(A^{*}, x\right)>-e\left(A^{*}\right), & x \in V V_{\mu / 6}^{-} .
\end{array}
$$


Define $W=\mid a, b] \sim\left[V_{\mu / 3}^{-} \cup V_{\mu / 3}^{+}\right]$. Let

$$
p_{1}=\sup \left\{E^{+}\left(A^{*}, x\right),-E^{-}\left(A^{*}, x\right): x \in W\right\},
$$

then $p_{1}$ is attained on compact $W$ and thus $p_{1}<e\left(A^{*}\right)$. Let

$$
p_{2}=\sup \left\{\left|f(x)-F\left(A^{*}, x\right)\right|: x \in \text { Frontier }(W)\right\},
$$

$p_{2}<e\left(A^{*}\right)$, and define $p=\max \left\{p_{1}, p_{2}\right\}$. There exists an extension of $f$ to $W$ such that $f$ is continuous on $[a, b]$, and

$$
\left|E\left(A^{*}, x\right)\right| \leqq p, \quad x \in W .
$$

By (5), (6), (7), $\left\|E\left(A^{*}, x\right)\right\|=e\left(A^{*}\right)$. Further $F\left(A^{*}, x\right)$ has $n$ alternations in the approximation of $f^{-}$and $f^{+}$if and only if $F\left(A^{*}, x\right)$ has $n$ alternations in the approximation of $f$. Now suppose that $F\left(A^{*}, x\right)$ does not have $n$ alternations. Define

$$
\begin{aligned}
& \nu^{+}=\sup \left\{-E^{-}\left(A^{*}, x\right): x \in C\left(V_{\mu / 3}^{+}\right)\right\}, \\
& \nu^{-}=\sup \left\{E^{+}\left(A^{*}, x\right): x \in C\left(V_{\mu / 3}^{-}\right)\right\},
\end{aligned}
$$

then $\nu^{\sigma}<e\left(A^{*}\right)$ since $C\left(V_{\mu / 3}^{\sigma}\right)$ contains no $-\sigma$ points. Let $\delta=(1 / 2)$ $\cdot\left[e\left(A^{*}\right)-\max \left\{p, \nu^{-}, \nu^{+}\right\}\right]$. By the argument of Tornheim [3, p. 464] there exists a parameter $A_{0}$ such that

and

$$
\left\|E\left(A_{0}, x\right)\right\|<\left\|E\left(A^{*}, x\right)\right\|=e\left(A^{*}\right)
$$

$$
\left\|F\left(A^{*}, x\right)-F\left(A_{0}, x\right)\right\|<\delta .
$$

The three following inequalities are direct consequences of earlier inequalities.

$$
\begin{aligned}
\left|E^{\sigma}\left(A_{0}, x\right)\right| & \leqq\left|f^{\sigma}(x)-F\left(A^{*}, x\right)\right|+\left|F\left(A^{*}, x\right)-F\left(A_{0}, x\right)\right| \\
& <p+\delta \leqq e\left(A^{*}\right)-\delta, \quad x \in W . \\
-e\left(A^{*}\right)+\delta & \leqq-\nu^{+}-\delta \leqq f^{-}(x)-F\left(A_{0}, x\right) \leqq E^{\sigma}\left(A_{0}, x\right) \\
& \leqq f(x)-F\left(A_{0}, x\right)<e\left(A^{*}\right), \quad x \in V_{\mu / 3}^{+} . \\
-e\left(A^{*}\right) \quad & <f(x)-F\left(A_{0}, x\right) \leqq E^{\sigma}\left(A_{0}, x\right) \leqq f^{+}(x)-F\left(A_{0}, x\right) \\
& \leqq \nu^{-}+\delta<e\left(A^{*}\right)-\delta, \quad x \in V_{\mu / 3}^{-} .
\end{aligned}
$$

Combining the three inequalities above, we have

$$
\left|E^{\sigma}\left(A_{0}, x\right)\right|<e\left(A^{*}\right), \quad x \in W \cup V_{\mu / 3}^{-} \cup V_{\mu / 3}^{+}=[a, b],
$$

hence $e\left(A_{0}\right)<e\left(A^{*}\right)$. Necessity of $n$ alternations for best approximations not having a straddle point has been shown and the theorem is proven. 
If $f^{-}$and $f^{+}$are continuous then the continuous function $f$ used in the proof of Theorem 1 can be selected to coincide with $f^{\sigma}$ on $V_{\mu / 3}^{\sigma}$ and we have the

Corollary. Let two continuous functions $f^{-}$and $f^{+}$have a best simultaneous approximation $F\left(A^{*}, x\right)$ with no straddle point. There exists a continuous function $f$ whose error $E\left(A^{*}, x\right)$ with respect to the same approximant coincides with the errors $E^{-}\left(A^{*}, x\right)$ and $E^{+}\left(A^{*}, x\right)$ in the neighbourhood of their absolute maxima and has no other absolute maxima. $F\left(A^{*}, x\right)$ is a best approximation to $f$.

3. Uniqueness of best approximations. To examine the uniqueness problem we need to consider double zeros, which are interior zeros at which a sign change does not occur. Variants of the following lemma are proven by Rice $[1$, p. $299 ; 2$, p. $57-58]$ for slightly different hypotheses on $F$.

LemMa 2. Let $F$ be an approximating function unisolvent of degree $n$, then the difference of two distinct approximations has less than $n$ zeros, counting double zeros twice.

Lemma 3. If $F\left(A^{*}, x\right)$ has $n$ alternations and $A_{0}, A^{*}$ are best, then $F\left(A^{*}, x\right)-F\left(A_{0}, x\right)$ has $n$ zeros, counting double zeros twice.

Proof. At a + point of $F\left(A^{*}, x\right), F\left(A_{0}, x\right)-F\left(A^{*}, x\right) \geqq 0$ and at a - point, $F\left(A_{0}, x\right)-F\left(A^{*}, x\right) \leqq 0$. Let $\left\{\mathrm{x}_{0}, \cdots, x_{m}\right\}$ be a set of ordered points on which alternation with respect to $F\left(A^{*}, x\right)$ takes place. On each interval $\left[x_{k}, x_{k+1}\right], F\left(A_{0}, x\right)-F\left(A^{*}, x\right)$ must have a zero. It is possible for two adjacent intervals to contain only one zero but it is readily seen by drawing a diagram that such a zero must be a double zero.

From Theorem 1, Lemma 2 and Lemma 3 follows immediately

Theorem 2. If $F\left(A^{*}, x\right)$ has $n$ alternations it is a unique best approximation to $f^{-}$and $f^{+}$on $[a, b]$.

In case only a straddle point occurs best approximations may not be unique. An example is given in the next section.

4. Related problems. Let us now consider approximation problems which can be reduced to the approximation problem studied in this note. First, suppose $f$ is a bounded function on $[a, b]$, then it is clear that the problem of minimizing $\|f(x)-F(A, x)\|$ can be replaced by the problem of approximation of the two functions $f^{-}, f^{+}$,

$$
f^{-}(x)=\liminf _{u \rightarrow x} f(u), \quad f^{+}(x)=\limsup _{u \rightarrow x} f(u),
$$


which differ from $f$ only at points of discontinuity. In case $f$ is continuous the best approximation has $n$ alternations and is unique (this is the classical case), whereas if $f$ is not continuous best approximations may have only one straddle point and not be unique. For example, let $f(x)=-1$ for $x \leqq 0, f(x)=1$ for $x>0$, then if $f$ is approximated by first degree polynomials on $[-1,1]$, all polynomials $a x$ for $0 \leqq a \leqq 2$ are best approximations and the point 0 is a straddle point.

Secondly, suppose $g(x, y)$ is a continuous function on a compact subset $S$ of 2 -space and we wish to find a parameter $A$ to minimize

$$
\sup \{|g(x, y)-F(A, x)|:(x, y) \in S\} .
$$

In case $\{x:(x, y) \in S\}$ is an interval we can define

$$
\begin{array}{lll}
g^{+}(x)=\sup \{g(x, y):(x, y) \in S\}, & g^{-}(x)=\inf \{g(x, y):(x, y) \in S\}, \\
f^{-}(x)=\liminf _{u \rightarrow x} g^{-}(u), & f^{+}(x)=\limsup _{u \rightarrow x} g^{+}(u) .
\end{array}
$$

A problem in multivariable Chebyshev approximation, for which very few results are known, has been reduced to a problem of approximation on an interval, for which many results are known.

Thirdly, the problem of simultaneous Chebyshev approximation of a finite number of continuous functions $f_{1}, \cdots, f_{m}$ is the problem of choosing a parameter $A$ to minimize

$$
\max \left\{\left\|f_{k}(x)-F(A, x)\right\|: k=1, \cdots, m\right\} .
$$

It suffices to define

$$
\begin{aligned}
& f^{+}(x)=\max \left\{f_{k}(x): k=1, \cdots, m\right\}, \\
& f^{-}(x)=\min \left\{f_{k}(x): k=1, \cdots, m\right\},
\end{aligned}
$$

and the problem of simultaneous approximation of $f^{-}$and $f^{+}$is an equivalent approximation problem.

\section{REFERENCES}

1. J. Rice, Tchebycheff approximation by functions unisolvent of variable degree, Trans. Amer. Math. Soc. 99 (1961), 298-302. 1964.

2. - The approximation of functions, Addison-Wesley, Reading, Mass.,

3. L. Tornheim, On n-parameter families of functions and associated convex functions, Trans. Amer. Math. Soc. 69 (1950), 457-467.

4. E. McShane and T. Botts, Real analysis, Van Nostrand, Princeton, N. J., 1959, 74-77.

University of Western Ontario, London, Canada 